\title{
Ultrasonographic Measurement of Placental Thickness During Pregnancy as a Parameter For Estimating Gestational Age of The Foetus
}

\author{
Khanal UP, Paudel S, Lohani B \\ Department of Radiology and Imaging, Tribhuvan University Teaching Hospital, Kathmandu, \\ Nepal \\ Received: January 10, 2016 \\ Accepted: May 01, 2016 \\ Cite this paper: \\ Khanal UP,Paudel S, Lohani B. Ultrasonographic Measurement of Placental Thickness During Pregnancy as a \\ Parameter For Estimating Gestational Age of The Foetus . Nepalese Journal of Radiology 2017;7(10):13-18.
}

\begin{abstract}
Introduction: Routine ultrasonography in pregnancy has the role in identifying the position, parenchyma echo pattern, variability of thickness of placenta which is associated with increased perinatal risk to both the foetus and mother. This study was carried out to establish the value of placental thickness measurement during normal pregnancy as a reliable parameter for estimating gestational age of the foetus.
\end{abstract}

Methods: This was a prospective cross sectional study done in the department of Radiology and Imaging at Tribhuvan University Teaching Hospital from September 2004 to April 2005. Total 350 normal pregnant women of more than 15 weeks of gestation were included. Ultrasonographic measurement of placental thickness and foetal biometric measurements were obtained. Correlation co-efficient tests were performed between the gestational age in weeks and placental thickness using Karl Pearson formula in SPSS 10 package.

Results: Mean placental thickness measured at the cord insertion site increased from 15.28 $\mathrm{mm}$ at 15 weeks of gestational age to $39.57 \mathrm{~mm}$ at 39 weeks of gestational age correlated almost exact with a standard mean of deviation of $\pm 1.8 \mathrm{~mm}(\mathrm{r}>0.851, \mathrm{p}<0.001)$.

Conclusion: Ultrasonographic measurement of placental thickness during normal pregnancy can predict the gestational age of the foetus with high confidence level and can be included as one of the biometric measurements of foetus to estimate its gestational age.

Key words: Gestational age, Placental thickness, Ultrasonography

Correspondence to: Umesh Prasad Khanal

Assistant Professor, Department of Radiology and Imaging

Institute of Medicine, Tribhuvan University

Teaching Hospital

Kathmandu, Nepal

E-mail: upkhanal2007@gmail.com

\section{INTRODUCTION}

The term placenta originated from Greek word plakuos meaning 'Flat cake'. The placenta is a materno-foetal organ which begins

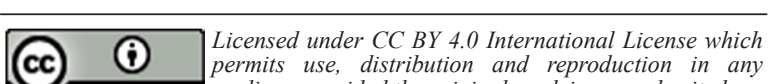
medium, provided the original work is properly cited
permits use, distribution and reproduction in any 
developing at implantation of the blastocyst and is delivered with the foetus after birth.

The placenta has the general form of a disc-like flattened cake moulded with the endometrial lining of the uterus. The mature has diameter of approximately $20 \mathrm{~cm}$ and about 3-4 cm thick centrally tapering towards edges. It weighs 500 grams when all blood is taken out.

The placenta is a foetal organ with important metabolic, endocrine and immunological functions besides being responsible for nutrition, respiration and excretion for the foetus. Acting as a barrier, it has a role in protecting foetus from noxious agents. Placental formation begins in the latter half of second month of pregnancy and usually completed by the fourth month. It reaches its maximum growth at term.

With new advances in the gray scale and Doppler sonography, we are able to study placental sonographic appearance and its relation to the uteroplacental blood flow measurement and intrauterine growth. Presently the most effective way to date the pregnancy is by the use of ultrasound. ${ }^{1-8}$

Several sonographically derived parameters used to date pregnancy include Crown rump length (CRL), Biparietal diameter (BPD), Head circumference (HC), Femoral length (FL) and Abdominal circumference (AC). Routine ultrasonography in pregnancy has the role in identifying the position, parenchyma echo pattern, variability of thickness of placenta which is associated with increased perinatal risk to both the foetus and mother. ${ }^{9}$ Placental thickness measured at the level of umbilical cord insertion can be used as a new parameter to estimate the gestational age of the foetus. The present study was undertaken to evaluate the relationship between placental thickness and gestational age of the foetus and compare the results obtained with the other studies carried out elsewhere.

\section{METHODS}

This was a prospective cross sectional study conducted in the Department of Radiology and Imaging with the help of the Department of Obstetrics and Gynecology at tertiary hospital in Kathmandu, Nepal. Study was conducted from the month of September 2004 to April 2005. The study comprised of 350 pregnant women at or above 15 weeks of pregnancy who attended the Obstetrics and Gynecology outpatient department for antenatal checkup. The participants of the study were explained in detail regarding the nature of the study and its benefits and only those who agreed to participate in the study were included.

Multiple pregnancies, pregnancy with intrauterine growth retardation of the foetus, foetus with congenital malformations identified during the time of scan, hydrops fetalis, patients with pregnancy induced hypertension and diabetes mellitus were excluded from the study.

Study was conducted using curvilinear array, real time, B-mode ultrasound equipped with a 3.5 and $5 \mathrm{MHz}$ frequency probes with Doppler facility in SONOACE 8800 MEDISONS Co. Patient was kept in supine position and trans abdominal ultrasonography was performed. The site of insertion of umbilical artery was identified by color Doppler study.

Ultrasonographic examination of placenta included the site localization, parenchymal echopattern and thickness. The placental thickness was obtained at the maximum vertical length at the level of cord insertion. Placental thickness was measured in millimeters.

Foetal biometric parameters were measured with screen calipers on the freeze pictures. Biparietal diameter (BPD), Head circumference (HC), Abdominal circumference (AC) and Femoral length (FL) 
were measured in millimeters using standard techniques (HADLOCK formula).

Permission was obtained from head of departments of Radiology and Imaging as well as Obstetrics and Gynecology of TUTH and protocol was approved by ethical board of the institution. Informed consent was obtained from all the participants after explaining the procedure.
Numerical data obtained was analyzed using statistical methods with the use of computer software, SPSS 10 package. The mean placental thickness for every week of gestation from 15 weeks till 39 weeks were obtained along with standard deviation and standard error of mean was calculated in each measurement. Co-relations of the gestational age with placental thickness were obtained by Pearson co-relation formula. Graph of the corelation was obtained and studied.

\section{RESULTS}

Table 1: Placental thickness at different gestational age

\begin{tabular}{|c|c|c|c|c|c|}
\hline \multirow[b]{2}{*}{ Week of Gestation } & \multirow[b]{2}{*}{$\mathbf{N}$} & \multicolumn{4}{|c|}{ Placental thickness (mm) } \\
\hline & & Minimum & Maximum & Mean & $\begin{array}{l}\text { Std. Devia- } \\
\text { tion }\end{array}$ \\
\hline 15 & 6 & 14.00 & 17.00 & 15.2833 & 1.1321 \\
\hline 16 & 7 & 16.00 & 19.00 & 17.0286 & 0.9499 \\
\hline 17 & 12 & 16.80 & 18.80 & 17.6417 & 0.6360 \\
\hline 18 & 15 & 17.00 & 19.80 & 18.5533 & 0.8991 \\
\hline 19 & 18 & 15.00 & 25.00 & 19.2444 & 2.1840 \\
\hline 20 & 14 & 16.00 & 25.00 & 19.8143 & 2.2305 \\
\hline 21 & 17 & 16.00 & 28.00 & 21.7941 & 2.6102 \\
\hline 22 & 15 & 21.00 & 27.00 & 23.0533 & 1.6155 \\
\hline 23 & 12 & 21.00 & 29.00 & 23.7330 & 2.0042 \\
\hline 24 & 10 & 21.00 & 25.00 & 23.9000 & 1.4491 \\
\hline 25 & 10 & 24.00 & 28.00 & 25.5800 & 1.3011 \\
\hline 26 & 10 & 23.00 & 29.00 & 25.7200 & 1.7242 \\
\hline 27 & 19 & 25.00 & 31.00 & 28.0000 & 1.7692 \\
\hline 28 & 14 & 25.50 & 31.00 & 28.6929 & 1.3691 \\
\hline 29 & 18 & 26.00 & 30.00 & 28.7389 & 1.1330 \\
\hline 30 & 16 & 29.00 & 31.80 & 30.1750 & 0.8250 \\
\hline 31 & 15 & 27.00 & 40.00 & 31.5800 & 2.8411 \\
\hline 32 & 11 & 30.50 & 37.00 & 32.4273 & 1.7333 \\
\hline 33 & 16 & 27.00 & 37.00 & 33.4500 & 2.0163 \\
\hline 34 & 19 & 30.40 & 35.00 & 33.9158 & 1.3074 \\
\hline 35 & 20 & 30.80 & 39.00 & 34.7500 & 1.8509 \\
\hline 36 & 14 & 31.00 & 40.50 & 36.8357 & 2.5239 \\
\hline 37 & 16 & 32.00 & 48.00 & 37.5375 & 3.6628 \\
\hline 38 & 14 & 34.80 & 45.00 & 39.1500 & 2.6958 \\
\hline 39 & 12 & 35.00 & 45.00 & 39.5750 & 2.9588 \\
\hline Total No of cases $=$ & 350 & & & & \\
\hline
\end{tabular}


In this prospective cross sectional study, sonographic measurement of placental thickness was compared with Gestational age of the foetus in each week of gestation from $15^{\text {th }}$ weeks to $39^{\text {th }}$ weeks. A total of 350 normal pregnant women were included.

The age of the subjects ranged from 17 years to 35 years with a mean age of $25.0 \pm 4$ years.

Placental thickness at various gestational ages were measured (Table 1, Fig. 1).
The maximum placental thickness measured was $48.5 \mathrm{~mm}$ and minimum was $14.0 \mathrm{~mm}$. mean placental thickness increased steadily from $15.28 \mathrm{~mm}$ at 15 weeks of gestation to $39.5 \mathrm{~mm}$ at 39 weeks of gestation. The standard deviation in placental thickness measurement also varied from $\pm 0.825 \mathrm{~mm}(\mathrm{p}<0.001)$ at 30 weeks to $\pm 2.958 \mathrm{~mm}(\mathrm{p}<0.001)$ at 39 weeks of gestation. The placental thickness of the foetus of $15^{\text {th }}$ week till 39 weeks of gestational age correlated almost exact with a standard deviation of $1.8 \mathrm{~mm}(\mathrm{r}>0.851, \mathrm{p}<0.001)$.

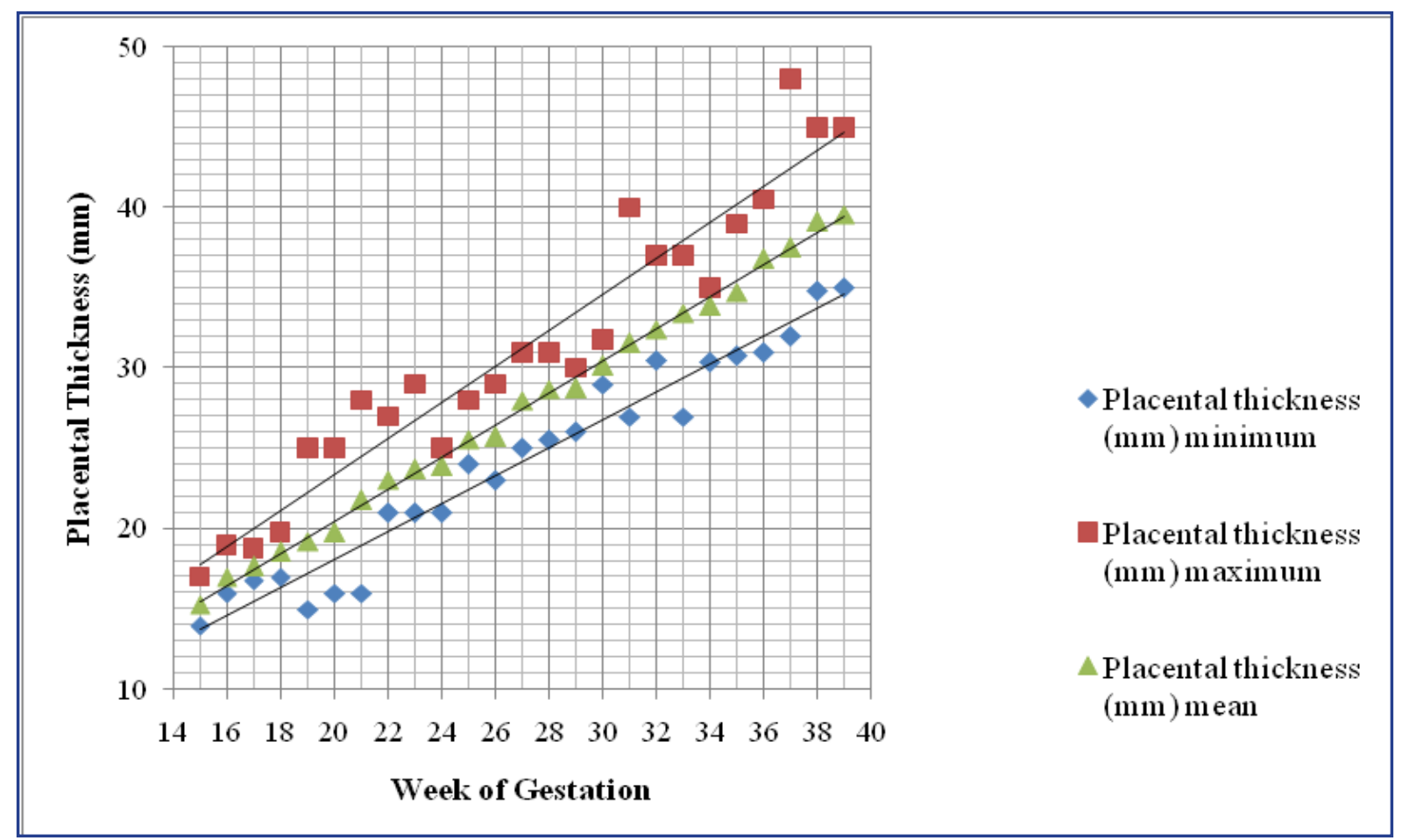

Figure 1: Mean placental thickness at different weeks of gestational.

\section{DISCUSSION}

It is well known that gestational age of the foetus can be estimated by measurement of various parts of the foetus. It has been in practice since 1970s when Robinson started using Ultrasonography for this purpose. The CRL, BPD, HC, AC and FL are routinely measured in obstetric sonography along with the morphology of various structures of the foetus to exclude any eventuality that may crop up during pregnancy. This has significantly reduced both morbidity and mortality of the foetus as well as the mother. ${ }^{9}$
The crown rump length (CRL) between 7 to 12 weeks is the most accurate parameter for first trimester dating. In the second trimester, the Biparietal diameter (BPD), head circumference (HC), transcerebellar diameter (TCD), abdominal circumference (AC), femoral length (FL) and long bones can be measured to estimate gestational age. $\mathrm{HC}$ is considered to be the better indicator than BPD as it takes account of various shapes of growing skull.

After 32 weeks the composite factors ( $\mathrm{HC}$, $\mathrm{AC}$ and FL) are accurate. However, the best 
assessment of foetal gestational age should be corrected with previous ultrasound findings.

The other parameters to date pregnancy include embryonic trunk circumference, orbital diameter, scapular length, thigh circumference and foot length. Serial study of all these give fairly approximate foetal gestational age.

In this prospective study the thickness of placenta was measured for each gestational week by ultrasound starting from $15^{\text {th }}$ week. The gestational week of the foetus was determined by BPD, FL, AC \& HC using the HADLOCK formula. There was strong positive correlation between placental thickness and gestational age in all weeks of gestation $(\mathrm{p}<0.001)$.

Similar study done by Mital P et al at SMS Medical College, Jaipur, India in 2002 on 600 antenatal women of all gestational age more than 10 weeks of gestation had revealed similar findings. From $22^{\text {nd }}$ weeks to the $35^{\text {th }}$ weeks of gestation, the placental thickness coincide almost exactly with gestational age in the weeks. ${ }^{10}$

In addition to gestational age assessment, placental thickness can be used in predicting intrauterine growth retardation. As demonstrated by Habib A Fawzia at department of obstetrics and gynecology, King Khalid University Hospital, Riyad, Saudi Arabia in 2002, measurement of placental diameter and thickness in all sonographic assessment of pregnancy in addition to the routine use of uterine artery Doppler in the second trimester helps in predicting low birth weight infants. ${ }^{10}$

\section{CONCLUSIONS}

There was strong positive correlation between the placental thickness at each week of gestational age of foetus to the gestational age of the foetus calculated by other parameters. The ultrasonographic measurement of placental thickness during normal pregnancy can predict the gestational age of the foetus with high confidence level ( $>95 \%$ at all gestational age). In cases where the exact duration of pregnancy is not known measuring the placental thickness alone could give important clue about it. Thus placental thickness at the insertion of umbilical cord as measured by ultrasonography is one of the biometric measurements of the foetus to estimate its gestational age.

We feel that further study involving larger number of normal pregnancies would be valuable.

\section{CONFLICT OF INTEREST}

None

\section{SOURCES OF FUNDING}

None

\section{REFERENCES}

1. Sutton D. Textbook of Radiology and Imaging. $6^{\text {th }} \mathrm{Ed}$. Churchill Livingstone 1998: p1208-1215.

2. Benson C.B \& Doubilet P.M. Sonographic prediction of gestational age: accuracy of second and third trimester foetal measurements. $\mathrm{Am}$ J Rad 1990;157(6):1275-1277. Available from: https://www.ajronline. org/doi/pdf/10.2214/ajr.157.6.1950881 [Accessed 10th December 2015].

3. Hadlock FP, Harrist R.B, Deter RL. Foetal femur length as a predictor of menstrual age: sonographically measured. Am J Rad 1982;138(5):875-878. https://doi.org/10.2214/ajr.138.5.875

4. Yeh MN, Barcero L, Reilly KB. Ultrasonic measurement of the femur length as an index of foetal gestational age. $\mathrm{Am} \mathrm{J}$ Obst \& Gynecol 1982;144(5): 519-522. https://doi.org/10.1016/00029378(82)90219-8 
5. Pretorius DH, Nelson TR, MancoJohnson ML. Foetal age estimation by ultrasound: The impact of measurement. Am J Rad_1984 Sep;152(3): 763-766. https://doi.org/10.1148/ radiology.152.3.6463258

6. Smith GN, Frey KA, Johnson TR. Assessing gestational age. Am Fam Physician $J$ 1986;33(2):215-220. Available from: https://www.ncbi. nlm.nih.gov/pubmed/3511646 [Accessed 15th December 2015].

7. Rose BL, Lamb EJ. Foetal age determination. J Obst Gynecol 1989 Feb:243-249.

8. Hadlock FP, Deter RL, Carpenter RJ . Estimating foetal age: Effect of head shape on BPD. Am J Rontgenol 1981 Jul:83-85 Available from: https://www.ajronline. org/doi/pdf/10.2214/ajr.137.1.83 [Accessed 12th December 2015].

9. Mital P. Placental Thickness- A Sonographic Parameter For Estimating Gestational Age of The Foetus. Ind $J$ Radiol Imag 2002; 12(4):553-554. Available from: http://www.ijri. org/text.asp?2002/12/4/553/28540 [Accessed 12th December 2015].

10. Habib A. Prediction of low birth weight infants from ultrasound measurement of placental diameter and placental thickness. Ann Saudi Med 2002; 22: 5-6. https://doi.org/10.5144/0256$\underline{4947.2002 .312}$ 